\title{
Virtual Class room using six sense Technology
}

\author{
${ }^{1}$ Meenakshi Gupta, ${ }^{2}$ Shruti Sharma \\ ${ }^{1,2}$ Assistant Professor, Maharaja Agarsen Institute of Management Studies, Affiliated to G.G.S.I.P.U.
}

\begin{abstract}
Sixth Sense is a revolutionary technology that can be used to access digital information in the world with wearable gestures. This paper focuses on various applications of six sense technology in educational purpose and how a virtual classroom, can be conducted anywhere, anytime with the help of six sense technology. Sometimes, It may happen professor or students has the limitations of not carrying the laptop. Therefore, with the help of six sense technologies, It will allow students, professors, researchers to interact with our world like never before. Six sense technology introduces the concept of online multimedia experience as various journals, articles can be accessed anywhere without having computer and without being connected to Internet .It can also recognize articles and newspaper, retrieve the latest related stories or video from the internet and then display them on pages for user. User may not necessary use dictionary or encyclopedia. We can get information on anything we want from anywhere within a few moments. Human figures can be made as keypad where students can perform all the calculations. Researchers' can capture the images without having camera.Human fingers works like the keyboard as well as the mouse. All the typed documents can be converted into digital notes It is a hi-tech device that allows users to be part of the physical world and still access the digital information existing on the World Wide Web by using natural hand gestures. Using Sixth Sense technology in class rooms will creates a more stimulating learning environment. The Sixth Sense prototype is comprised of a pocket projector, a mirror, colored marker and a camera. These components are coupled in a pendant like mobile wearable device. The camera, mirror and projector is connected wirelessly to a blue tooth smart phone device that can easily fit into the user's pocket. The key here is that sixth sense recognizes the objects around you and displaying the information automatically and letting you access it in any way you want.
\end{abstract}

\section{Introduction}

Integration and collaboration of six sense technology in the classrooms will be a new concept over the virtual classrooms and online teaching concept. As virtual class rooms have the limitations of expensive hardware setup, this concept will help to establish the classrooms without computers, desktops, projectors etc. Infact This classroom structure can be setup without the need of internet. With six sense technology, Students without having the laptops and without being connected to internet, can do the research, can get information about any topic within the fraction of seconds. Information is basically confined traditionally on paper or digitally on screen. Six sense bridges this gap, bringing intangible digital information out into the tangible information. and allowing us to interact with them with the help of hand gesture,. Six sense technologies is a wearable gesture based device that augments the physical world with the digital information and let's people use natural hand gestures to interact with the information. It was developed by Pranav Mistry, a Phd Student in the fluid interfaces Group at MIT laboratory; he waved the curtain of six sense technology. It involves the reception of information not gained through any of our five senses, It works besides the five senses. Infact six sense technology is the blend of computer, internet, camera and mobile phone. It works as the wearable device that is hanged around the neck of a person and thus the projection starts by the mean of micro projector attached to device. It is based on the concepts of augmented reality and has well implemented the perceptions of it. Sixth sense technology has integrated the real world objects with digital world. The fabulous 6th sense technology is a blend of many exquisite technologies. The thing which makes it magnificent is the marvelous integration of all those technologies and presents it into a single portable and economical product Future generations will likely marvel at the fact that we had to sit in classrooms with physical books and listen, in person, to a teacher lecture etc. The device sees what we see but it lets out information that we want to know while viewing the object. It can project information on any surface, be it a wall, table or any other object and uses hand / arm movements to help us interact with the projected information. The device brings us closer to reality and assists us in making right decisions by providing the relevant information, thereby, making the entire world a computer. The Sixth Sense prototype implements several applications that demonstrate the usefulness, viability and flexibility of the system. The map application lets the user navigate a map displayed on a nearby surface using hand gestures, similar to gestures supported by Multi-Touch based systems, letting the user zoom in, zoom out or pan using intuitive hand movements The user can stop by any surface or wall and flick through the photos he/she has taken. Sixth Sense also lets the user draw icons or symbols in the air using the movement of the index finger and recognizes those symbols as interaction instructions. For example, drawing a magnifying glass symbol takes the 
user to the map application or drawing a '@' symbol lets the user check his mail. The Sixth Sense system also augments physical objects the user is interacting with by projecting more information about these objects projected on them.

\section{Working Of Six Sense Technolgy}

The hardware structure of the six sense technology contains various components in which all the components are coupled in pendant like mobile wearable device such as

- Camera

- Projector

- Mirror

- Mirror component

- Color Markers

The hardware structure of the six sense technology comprises of a wearable pendant, mirror, camera, and projector and is connected wirelessly to Bluetooth or $3 \mathrm{~g}$ or wifi Smartphone's that can be easily carried out in human's pocket. It has a camera that recognizes individuals, images, pictures, gestures that one make with their hands by gesture recognition. After the image is capture, it is send to smart phones for further processing. The downward projector works like a screen which projects output data and images.

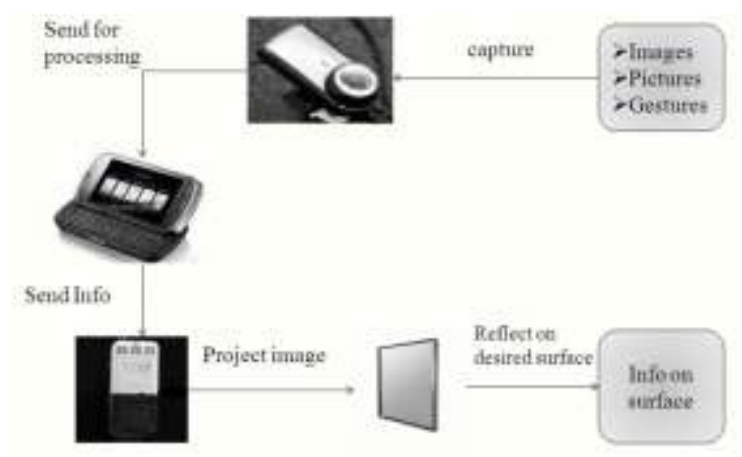

Fig 2.1 Working of Six Sense Technology

\subsection{Head Mounted Display}

\section{Technology Behind The Sixth Sense}

A head mounted display (HMD) places images of both physical world and registered virtual graphical objects over the user's view of world. The HMD are either optical or video see through in nature.

\subsection{Hand Held Display}

Hand Held display employs a small computing device with the display that fits into the hand. All hands Augmented Reality (AR) solutions till date have employed video see through technologies which display graphical information to the outside physical world.

\subsection{Gesture Recognition}

It is a concept and language technology with the goal of interpreting human gestures via mathematical algorithms. Gestures mainly instigate from body motion in which computers understand the body movements. Gesture recognition limits the inputs to computers from keyboard, mouse and other input devices; and enables to interface with computer without any devices e.g. using finger to move the pointer on computer screen. Gesture recognition can be conducted using computer vision and image processing techniques

In computer vision the computer can extract the information from an image that is necessary to solve a specific task. The image data can take many forms, such as videos, views from multiple cameras, or multidimensional data from a medical scanner. As a technological discipline, computer vision seeks to apply its theories and models to the construction of computer vision systems. Examples of applications of computer vision include systems for: Controlling processes, Detecting events, Organizing information, Modeling objects or environments. Computer vision is closely related to the study of biological vision. The field of biological vision studies and models the physiological processes behind visual perception in humans and other animals. Computer vision, on the other hand, studies and describes the processes implemented in software and hardware behind artificial vision systems 


\subsection{Radio Frequency Identification}

Six sense technologies is a platform used for radio frequency identification with information from other enterprise systems and sensors to automatically make inferences about people, their objects, workstations and their interactions. In radio frequency identification two devices are tagged to communicate with each other through radio waves.

\subsection{Hardware Required}

\section{Hardware Requirnments}

- 4.1.1. Mobile

- Nokia n95 smart phone is used (running symbian O.S s60edition).

- It has multitasking capability.

- Built-in camera provides execution of both:

- Gesture tracking engine.

- Gesture enabled application.

\subsubsection{Projector}

- Pocket projector Pk101 from Optima is used.

- It augments nearby surfaces.

- It is a LED based projector.

- Suitable for mobile usage

\subsubsection{Software Required}

- Applications are simple or video mented using JAVA 2MICROedition.

- Computer vision library is written in Symbian c++ (used in Gesture tracking).

- The software for the sixth sense prototype is developed on a Microsoft Windows platform using C\#, WPF

- open CV

\subsection{Zoom In/Zoom Out}

\section{Application}

This is the basic feature used by the students while doing case study. The miniature content of the books can be magnified to focus more light on it. The user can zoom out or zoom in using intuitive hand movements. By the use of this technology, user can focus on deep study of the articles without using computer. Secondly, pictures can be magnified and contents can be deeply visualized.

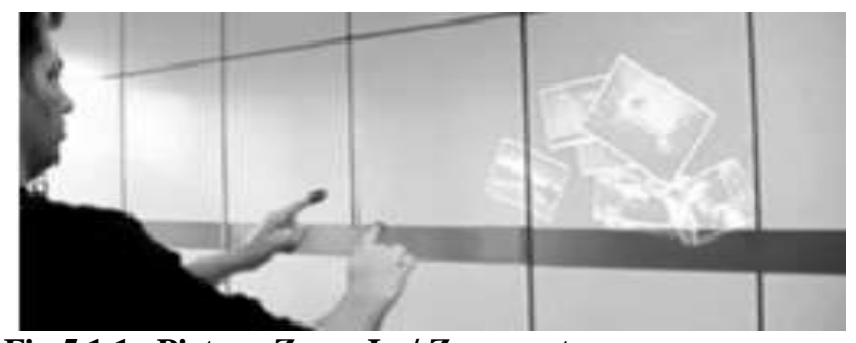

Fig 5.1.1 Picture Zoom In / Zoom out

\subsection{Calculations and Data Analyze}

With the help of six sense technology, a keypad can be projected on our palm where the numbers will be available on the finger. This keypad looks like a calculator and contains all the numeric buttons. Secondly students may not need to carry Mobile Device or calculator along with themselves

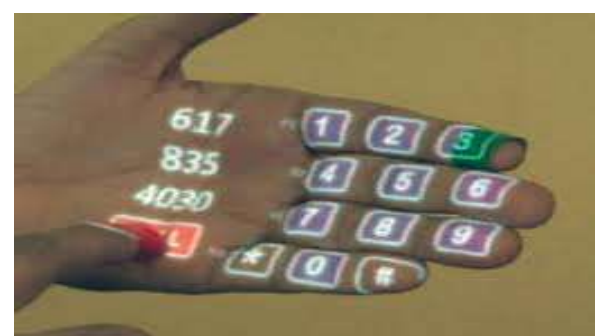

Fig 5.1.2 Calculator Key pad on fingers 
all the time. The key will come on the one hand fingers whereas the other hand fingers will $b$ bused to select the keys. This is very beneficial for the account students.

\subsection{Creating Multimedia Online Experience}

This is the most beneficial segment of six sense technology as a piece of paper can be easily converted into paper. For example, while reading books, journals, newspaper. While reading the content, more live information can be projected on a particular topic without being connected to internet .The device can also recognize articles and newspaper, retrieve the latest related stories or video from the internet and then display them on pages for user. User may not necessary use dictionary or encyclopedia. For Book Lovers, it is like a blessing and miracle, whenever we open the book, with the help of this technology, we can convert readable text into audible and much more information about that particular text.

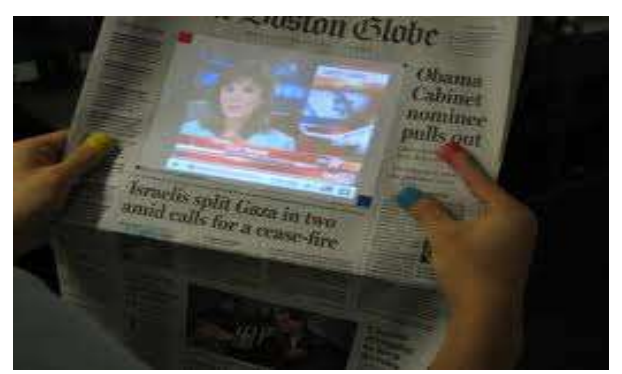

Fig 5.1.3 Online News Paper

\subsection{Video Conferencing and call making}

Six sense technologies can be used to make a call by projecting keypad on our fingers as shown in figure above. No mobile phone will be required to make a call. The number can be dialed simply without carrying cell phones

\subsection{Taking Pictures}

Sometimes it happens that student may need to capture the images while travelling so that they can be used for the future use and study about them. For this, It is not necessary to carry cameras, rather picture can be captured and saved by using six sense technology. If we fashion our fingers as shown in the fingers for any particular snapshot, the image of that particular area will be captured. Infact After taking the desired number of pictures, we can project them onto the surface and use our hand gesture to sort through the photos, organize and resize them. If this technology does nothing else. The idea is that the students can simply use their hands to draw images on the wall and take picture with their hands can be used laterally.

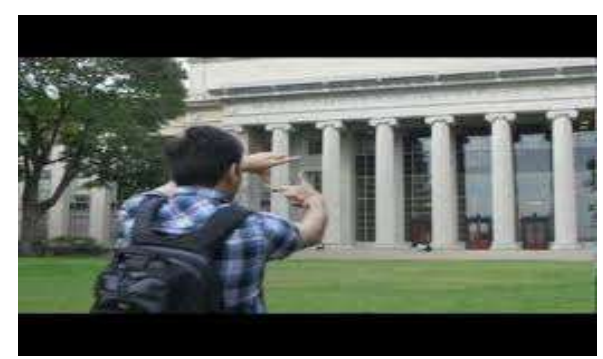

Fig 5.1.4 Fingers projected camera

\subsection{Getting Information about students Results}

Students can get the various information's such as admit cards, result, online submission status, project status etc using six sense technology without being connected to internet. It helps to get the information about anything instantly.

\subsection{Virtual Environment}

This facility enables user to create computerized environment anywhere, any time without having the real components such as having mouse on the table. The table top can be converted into computer screen. User can create computer lab anywhere without having all the input and output devices. 


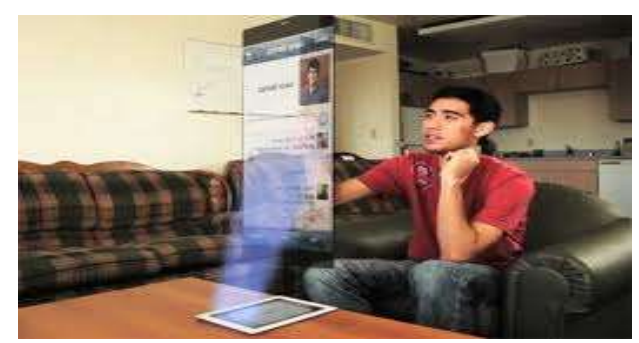

Fig 5.1.5 Computer Friendly Environment

\subsection{Magical Teaching White Board}

Black and white teaching boards are outdated infact smart board is latest these days and are very adaptable also. But when we have to teach something on smart board, so we have to connect it to our laptops, notebook so that internet can be accessed. But integrating smart board with six sense technology will be a big benefit for the academicians

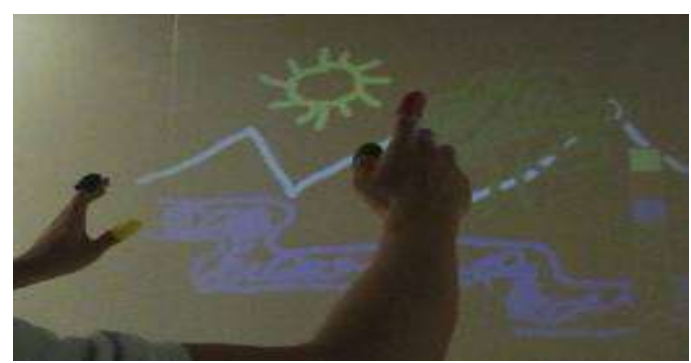

Fig 5.1.6 3D Drawing, Magical Board

\subsection{DIGITAL NOTES}

With the six sense technology, a dummy hand written document can be simply transferred into interactive document. While reading the notes, their content can be modified, altered with more content by converting them to digital notes as shown in figure.

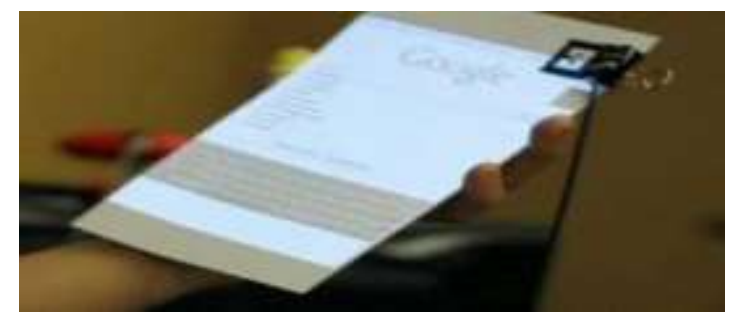

Fig 5.1.7 Digital Notes

VI. Advantages

- Sixth Sense is a wearable component which can be wearing as ornaments on human body. So there is no fear of losing it or misplacing it. It automatically becomes highly portable technology.

- It connects human being to the entire world without being connected to internet. It is an open source and cost effective and a person can map up an idea anywhere.

- It is very easy to adapt. It supports multi-touch and multi user interaction. This technology can be used by anyone without having the basic knowledge of keyboard or mouse.

- It will reduce the cost of setting up a classroom as teachers will not be required to carry laptops, projectors.etc

- There is no need to carry a camera anymore. If we are going for a holiday, then from now on wards it will be easy to capture photos by using mere fingers.

\section{Disadvantages}

- This technology has to all the time with human body parts. So high waves radiation emitted may cause severe diseases.

- It requires charging devices and the complete kit has to be recharged at continuous intervals. This cannot be used for long period of time as it has less battery back up 


\section{Future Conclusion}

Collaboration of six sense technology in the classrooms will introduce revolutionary change. This technology will create better understanding and level of interaction between the students and teachers will improve. It will also save the students, teachers, professors and researchers' time spent in huge research work and various other activities. It is also going to introduce the concept of automatic conference booking which will be a great help in group discussions, forums etc where students and teachers can interact with their ideas .Teaching will be more interesting and will be possible anywhere. Infact, this new concept can make online teaching more easy to adapt and convenient. Research work and detailed studies about any topic will be done in a short span of time. Information would literally be at students fingertips, as if it isn't already but even more so with this new invention. I could imagine students being engaged and fully interacting with their environment and learning, as if they are in a museum. Anywhere could be a learning environment, and the classroom itself would open up. The walls would no longer be barriers to the outside world. There may be information overload. Besides education, six sense technologies have many other benefits also in the real time environment. For example, there comes a situation many a times when we are shopping or in the mall and are not sure which brand to get. It is helpful in product listing and selection when we are purchasing any new product.

Annotating Video with Physical Events will be other future aspect in which six sense technologies will record the video feed from a camera with RFID events corresponding to inter-zone movement and object interaction. The video could, for instance, be from the security cameras in a building. With the video thus annotated with physical events, a user who is searching for a misplaced objected but suspects that it went missing in the lobby could issue a query like: "find me all segments of the security video recording corresponding to times when I passed through the lobby". This would then return the desired segments of video, relieving the user of the burden of sifting through hours of irrelevant video recording.

\section{References}

[1] (INCOCCI), 2010 International Conference, 27-29 Dec. 2010, Rao, S.S. Electron. \& Communication. Eng., Anna Univ. of Technol., Coimbatore, India ,336-339, Conference Publications

[2] Vsrd International journal of computer sceince \& InfomartionTechnology,VSRD-IJCSIT, Vol. 2 (2), 2012, 137-14

[3] International Journal ofInformation Technology and Knowledge Management. January-June 2012, Volume 5, No. 1, pp. 201-204

[4] Lenin Ravindranath, venkat padmanabhan, and piyush agrawal, SixthSense: RFID-based Enterprise Intelligence, in ACM Mobisys, Association for Computing Machinery, Inc., June 2008

[5] http://en.wikipedia.org/wiki/Gesture_recognition

[6] http://gizmodo.com/5167790/sixth-sense-technology-may-change-how-we-look-at-the-world forever

[7] http://theviewspaper.net/sixth-sense-technology-will-revolutionize-the-world/ 